\title{
Studies of Interlayer Magnetic Coupling in All-Semiconductor Superlattices by Means of Neutron Scattering Techniques
}

\author{
H. $\operatorname{KEgPA}^{a, b}$ AND T.M. GiebULtowicz ${ }^{b, a}$ \\ ${ }^{a}$ Institute of Experimental Physics, Warsaw University \\ Hoża 69, 00-681 Warszawa, Poland \\ ${ }^{b}$ Physics Department, Oregon State University, Corvallis, OR 97331, USA
}

An overview of neutron scattering studies of ferromagnetic and antiferromagnetic all-semiconductor superlattices is presented. Diffraction experiments on MnTe/CdTe, MnTe/ZnTe and EuTe/PbTe superlattices show pronounced correlations between the MnTe and EuTe layers across the non-magnetic spacers, even though these layers are antiferromagnetic and the systems are nearly-insulating. Current theory status of these systems is discussed. Diffractometry and reflectometry data from EuS/PbS superlattices reveal pronounced antiferromagnetic coupling between the ferromagnetic EuS block. First polarized neutron reflectometry data from superlattices prepared of a novel ferromagnetic "spintronics" material, $\mathrm{Ga}(\mathrm{Mn}) \mathrm{As}$, are also presented.

PACS numbers: 75.25.+z, 75.50.Pp, 75.75.+a

\section{Introduction}

Recent successes in synthesizing a new generation of epitaxial ferromagnetic (FM) semiconductors based on the III-V compounds open broad opportunities for new studies $[1,2]$. The characterization of microscopic mechanisms underlying the ferromagnetic nature of these materials is a task of considerable current importance [3]. One effect that may offer much insight into long-range exchange interactions between the Mn ions in these systems is the recently observed [1] magnetic coupling between two Ga(Mn)As films separated by up to $30 \AA$ of pure non-magnetic GaAs. Such a range of interlayer coupling is typically seen in binary superlattices (SLs) composed of ferromagnetic/non-magnetic metals. It is now firmly established that in metallic systems the exchange transfer across the 
non-magnetic spacer is mediated by conduction electrons [4, 5]. The fact that in $\mathrm{Ga}(\mathrm{Mn}) \mathrm{As}$ the carrier concentration attains values close to those in typical metals suggests that in the $\mathrm{Ga}(\mathrm{Mn}) \mathrm{As} / \mathrm{GaAs} / \mathrm{Ga}(\mathrm{Mn})$ As trilayers the coupling is also conveyed by a carrier-assisted mechanism. It should be noted, however, that magnetic coupling across spacers thicker than $30 \AA$ was observed in other all-semiconductor multilayered structures in which the density of carriers was several orders of magnitude lower than in metals [6-12]. Although not all results from those systems are yet clearly understood, the experimental facts provide strong evidence for the existence of mechanisms capable of transferring magnetic interactions across thick layers of non-magnetic semiconductors without the involvement of mobile carriers. Those observations from the systems made of "first-generation" magnetic semiconductors are certainly of interest in the context of studies conducted on the novel FM materials.

In this paper, we present an overview of the existing results of interlayer coupling studies in all-semiconductor SLs. Experimental search for such effects was started in the early 1990s. At that time the systems available for experimentation were made exclusively from antiferromagnetic (AFM) semiconductors (EuTe, $\mathrm{MnTe}$ ). The only research tool capable of detecting correlations between AFM layers is neutron diffraction. Hence, this method played a key role in the early studies of semiconductor SLs. Recently, a successful technology of fabricating EuS/PbS SLs has been developed [13]. It made possible the use of neutrons for investigating correlations between FM semiconducting layers. One can, in principle, detect such correlations by other methods (e.g., SQUID magnetometry). Yet, neutron tools - conventional diffractometry and the technique of neutron reflectometry which is particularly well suited for studying thin FM layers - still play a leading role in this research, as they offer a more direct insight into the correlation than other experimental methods. The application of polarization analysis may even further strengthen the potential of neutron scattering techniques. An illustration for that may be the latest polarization-analysis reflectometry studies of $\mathrm{Ga}(\mathrm{Mn}) \mathrm{As} / \mathrm{GaAs}$ superlattices [14], also discussed in this article.

In Sec. 2 of this paper it is explained in a concise form how neutron techniques are used for probing interlayer magnetic correlations. An overview of the existing experimental results from four different all-semiconductor SL systems and a brief description of related model studies are presented in Sec. 3. This material is followed by a short discussion and closing comments in Sec. 4.

\section{Neutron scattering tools}

There are two powerful neutron scattering techniques that can be used for studying magnetic superlattices: conventional ("wide-angle") diffractometry and neutron reflectometry. In diffraction regime, the neutrons directly probe the correlations between individual magnetic spins in the scattering system. Therefore, 
this method can be used for investigating any type of magnetic order in a crystal - FM, AFM, or any other more complicated arrangement. Similarly, if the scattering system consists of larger "blocks" of ordered spins (e.g., of magnetically ordered layers in a superlattice structure), neutron diffraction is sensitive to correlations between such blocks. As an illustration it is instructive to consider a simple example. Let us suppose that a superlattice is made up of alternating magnetic and non-magnetic layers, consisting, respectively, of $m$ and $n$ atomic monolayers (Fig. 1). The magnetic atoms have only two Ising-like spin states, "up" $(p=1)$ and "down" ( $p=-1)$. The scattering intensity $I(\boldsymbol{Q})$ for such a system can be obtained using a standard equation of diffraction theory [15]:

$$
I(\boldsymbol{Q}) \propto\left|f(Q) \sum_{j}^{\text {all atoms }} p_{j} \mathrm{e}^{\mathrm{i} \boldsymbol{Q} \cdot \boldsymbol{r}_{\mathrm{j}}}\right|^{2}
$$

where $\boldsymbol{Q}$ is the wave vector transfer, $f(Q)$ is the magnetic form factor, and $\boldsymbol{r}_{j}=$ $\left(x_{j}, y_{j}, z_{j}\right)$ is the position of the $j$-th magnetic atom. In studies of superlattices the relevant $Q$-scans are those parallel to the growth axis ( $z$ ), so that in $Q$ only $Q_{z} \neq 0$. Thus the exponential function argument becomes $\mathrm{i} Q_{z} z_{j}$, which means that the spin position within its host monolayer does not matter. Hence, summing over individual spins can be replaced by summing over spin monolayers, and the sum $\sum p_{j}$ for a given monolayer $k$ can be replaced by its net magnetization $M_{k}$. The equation can be further modified by taking advantage of the superlattice periodicity and factoring out the sum corresponding to the "unit cell" in the multilayered structure (called the "single-layer structure factor" and denoted as $\left.F_{\text {s.l. }}\left(Q_{z}\right)\right)$. Now the sum is over the $l=1, \ldots, N$ magnetic layers in the $\mathrm{SL}$ chain

$$
\begin{gathered}
I\left(Q_{z}\right) \propto\left|f\left(Q_{z}\right) \sum_{k=1}^{m} M_{k} \mathrm{e}^{\mathrm{i} k d Q z} \sum_{l}^{N} P_{l} \mathrm{e}^{\mathrm{i} l D Q z}\right|^{2} \\
=f^{2}\left(Q_{z}\right)\left|F_{\text {s.l. }}\left(Q_{z}\right)\right|^{2}\left|\sum_{l}^{N} P_{l} \mathrm{e}^{\mathrm{i} l D Q z}\right|^{2},
\end{gathered}
$$

where $d$ is the spacing between monolayers, $D=(m+n) d$ is the SL period. The $P$ l coefficient is +1 if the spin configuration in the $l$-th layer is the same as in the $l=1$ layer, and -1 if it is reversed (see Fig. 1).

The structure factor $F_{\text {s.l. }}\left(Q_{z}\right)$ describes the peak profile that would be obtained by measuring diffraction from a single layer. It has the shape of a broad maximum accompanied by weak subsidiary maxima (the dashed curves in Figs. 2a and b). For ferromagnetic layers the main maxima occur at $Q_{z}=(2 \pi / d) \xi$ points $(\xi=1,2,3, \ldots)$, and for antiferromagnetic ones at $Q_{z}=(2 \pi / d) \eta$ points $(\eta=$ $\left.\frac{1}{2}, \frac{3}{2}, \frac{5}{2}, \ldots\right)$ - i.e., at the same positions where Bragg peaks would occur for bulk crystals with the same spin structure. 


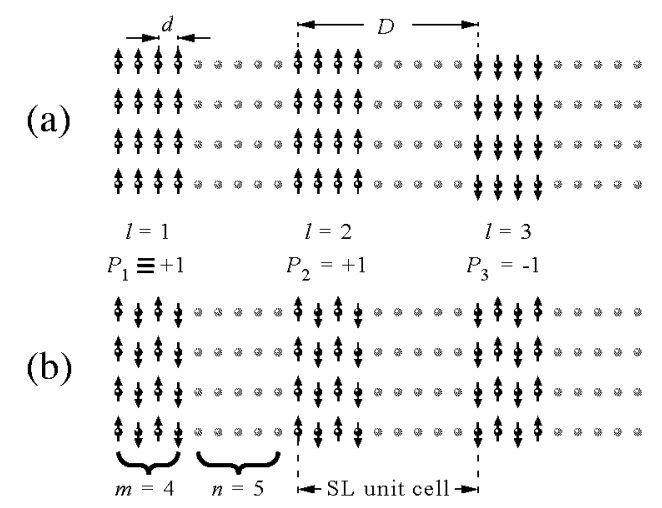

Fig. 1. Simple models of superlattices with Ising-like magnetic atoms and (a) ferromagnetic and (b) antiferromagnetic layers. Layers $l=1$ and 2 in the chain (a) are ferromagnetically correlated, and layers 2 and 3 are antiferromagnetically correlated. The AFM layers 1 and 2 are positively correlated, and 2 and 3 are "anticorrelated". The meaning of the symbols used in the text is explained in the graph.

The right-side squared modulus expression in Eq. (2) can be written as the sum of "self-correlation" and "layer-layer correlation" terms

$$
\sum_{l}^{N} P_{l} \mathrm{e}^{\mathrm{i} l D Q z}=N+\sum_{k \neq l} P_{k} P_{l} \mathrm{e}^{\mathrm{i}(k-l) D Q_{z}} .
$$

If there are no interlayer correlations, then the $P_{l}$ coefficient for successive layers takes the value of +1 or -1 in a random fashion - and, for large $N$, the layer-layer correlation term disappears on statistical averaging and $I\left(Q_{z}\right) \propto N^{2} f^{2}\left(Q_{z}\right)$ $\times\left|F_{\text {s.l. }}\left(Q_{z}\right)\right|^{2}$. Since $f(Q)$ is a slowly varying function, the spectrum has essentially the same shape as the squared single-layer structural factor.

If there are interlayer correlations in the system, the spectrum shape depends on the type of order in the layers (FM or AFM) and on how they are correlated. FM layers can be correlated either "ferromagnetically" (net magnetization always in the same direction) or "antiferromagnetically" (the magnetization sequence in successive layers is up-down-up-down...). In the former case all the $P_{l}$ coefficients have the same sign, and the rightmost squared sum in Eq. (2) becomes $\sin ^{2}\left(\frac{1}{2} N D Q_{z}\right) / \sin ^{2}\left(\frac{1}{2} D Q_{z}\right)$. This function has a sequence of sharp maxima at $Q_{z}=2 \pi n / D$ points $(n=0,1,2, \ldots)$. These maxima are "enveloped" by the $\left|F_{\text {s.l. }}\left(Q_{z}\right)\right|^{2}$ function, which results in a spectrum shape shown as the solid-line curve in Fig. 2a. For AFM interlayer correlations, on the other hand, the $P_{l}$ coefficients are +1 for odd $l$ 's, and -1 for even $l$ 's, and now the squared sum in Eq. (2) becomes $\cos ^{2}\left(\frac{1}{2} N D Q_{z}\right) / \cos ^{2}\left(\frac{1}{2} D Q_{z}\right)$, which has maxima at $Q_{z}=2 \pi\left(n+\frac{1}{2}\right) / D$ points and produces a spectrum shape as shown in Fig. 2b. Let us note that for FM interlayer correlations there is a central peak at the Bragg point with symmetric pairs of "satellites", whereas in the case of AFM correlations there is an inten- 
sity minimum at the Bragg position in between two "fringes" with equal heights. Such a clear difference in the spectrum shapes enables an easy identification of the correlation type.

In the case of AFM layers the rules are less straightforward. First, let us note that such layers may have a zero net moment (for even $m$ values), and then it does not make sense to describe the interlayer correlation as "FM" or "AFM". A better choice seems to be "positive correlations" if the monolayer magnetization sequence is the same in all layers, and "anticorrelations" if the sequence in the $k$-th layer is reversed in the $(k+1)$-th layer. It can be readily checked that the spectrum shape shown in Fig. 2a occurs in the case of positive correlations when $(m+n)$ is an even number, and anticorrelations when $(m+n)$ is an odd number; in other cases, the spectrum has the profile depicted in Fig. 2 b.

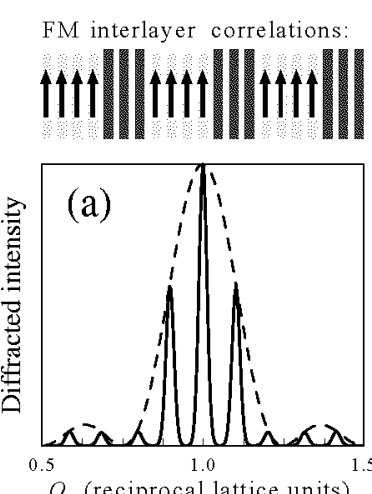

$Q_{z}$ (reciprocal lattice units)
AFM interlayer correlations:
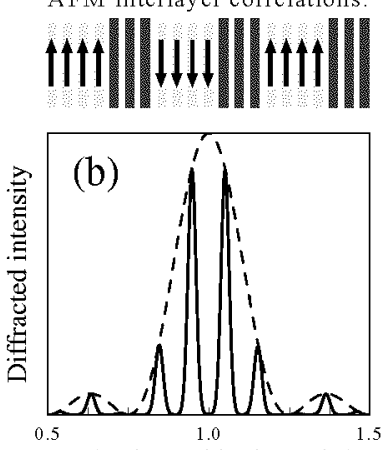

$Q_{z}$ (reciprocal lattice units)

Fig. 2. $Q_{z}$ diffraction profiles for: (a) a superlattice with FM correlations between FM layers, (b) a one with AFM correlations between FM layers. The spectra were calculated for model superlattices depicted at the top of each part. The dashed curve plotted in both parts is the shape of the squared single-layer structure factor $\left|F_{\text {s.l. }}\right|^{2}$, which is also the diffraction profile for a perfectly uncorrelated superlattice.

Neutron reflectometry. The refractive index $n$ of most condensed matter systems for neutron waves is slightly less than 1 (by $\approx 10^{-6}$ ), so that neutrons impinging a flat surface at a grazing angle $\theta$ lower than the critical angle $\theta_{c}=\sqrt{2(1-n)}$ are totally reflected [15]. The reflectivity $R(\theta)$ just above $\theta_{c}$ is a rapidly decreasing function. If there is a superlattice structure made of two materials with different refractive indices deposited on the reflecting surface, the $R(\theta)$ characteristic additionally exhibits sharp maxima at $\theta$ values satisfying the Bragg equation $\eta \lambda=2 D \sin \theta$ (where $\lambda$ is the neutron wavelength, $D$ is the SL period, and $\eta=1,2,3, \ldots$ ) [16]. In a magnetized FM material the interaction between the neutron magnetic moment $\mu_{\mathrm{N}}$ and atomic momenta gives rise to an additional term $\pm \mu_{\mathrm{N}} B / 2 E$ in $n$, where $B$ is the magnetic field within the material, $E$ is the neutron energy, and the sign depends on the orientation of the neutron spin relative to $\boldsymbol{B}[15,16]$. This enables one 

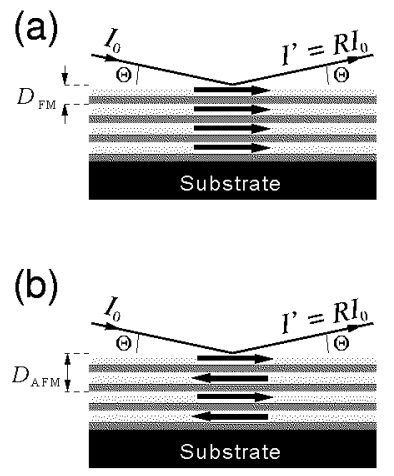
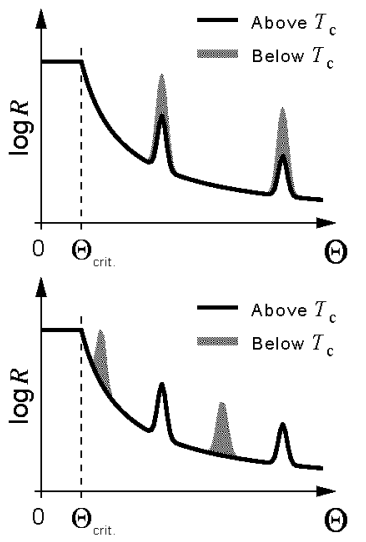

Fig. 3. Reflectivity profiles from a multilayered structure with: (a) FM, and (b) AFM correlations between ferromagnetic layers. The maxima in the solid curve are the structural Bragg peaks, and the shaded profiles show the positions of the magnetic peaks arising below $T_{\mathrm{C}}$.

to determine the type of interlayer correlations in FM superlattices. If the layers are ferromagnetically coupled, the neutrons "see" a magnetic superlattice which has the same periodicity as the atomic structure, and the magnetic peaks occur at the same positions as the structural ones (Fig. 3a). An AFM coupling effectively doubles the magnetic periodicity, and the peaks occur half-way in between the structural ones (Fig. 3b). It should be noted that the intensity and resolution in reflectometry is considerably better than in diffraction experiments. However, this method cannot be used for studying AFM layers in which $\boldsymbol{B}$ is always zero due to the zero net moment.

\section{Overview of experimental and model results}

\subsection{II-VI based systems}

II-VI/Mn-VI superlattices were the first magnetic semiconductor epitaxial structures investigated by neutron techniques $[9,17,18]$. In these epitaxial systems the magnetic constituents, MnSe and MnTe, crystallize in the metastable zinc blende (ZB) structure, not found in bulk. The ZB modifications are strongly frustrated fcc antiferromagnets that at low temperatures form the so-called type III AFM.

Pronounced interlayer coupling effects were observed in two II-VI/Mn-VI structures, CdTe/MnTe and ZnTe/MnTe with [001] growth axis [6-8]. Although the chemical formulae are very similar, these two systems are magnetically quite different. This comes from the fact that the type III AFM order in the frustrated spin lattices is very sensitive to symmetry-breaking strains.

The unstrained cubic lattice parameter of CdTe is larger than that of ZB MnTe. Therefore, in the CdTe/MnTe structures the MnTe layers are stretched- 
i.e., the lattice period $a_{x y}$ along the [100] and [010] in-plane axes is elongated, while the period $a_{z}$ in the [001] growth direction is shortened $\left(a_{z}<a_{\mathrm{MnTe}}<a_{x y}\right)$. Such a lattice distortion causes a transition from the commensurate type III order to a new structure in which the Mn spin directions are arranged in a helical fashion [17]. The axis of the spin helix is parallel to one of the in-plane axes, and the helix pitch is incommensurate with the atomic lattice period.

A theoretical explanation of the effects seen in the CdTe/MnTe has been recently proposed by Rusin [19] who pointed out that even though there are no mobile carriers in the system at low $T$, CdTe does contain carriers that are bound by impurities or defects, forming "hydrogenic centers" with the Bohr radius of several tens of $\AA$. In diluted magnetic semiconductors such centers may polarize magnetic ions within the Bohr orbit, giving rise to an effect known as "bound magnetic polaron" (BMP). Thus in the Rusin model the centers located in the spacer act in a similar way on the interface Mn spins from the two adjacent MnTe blocks, "synchronizing" their polarization and thus effectively introducing magnetic correlations between the spin helices.

In contrast to $\mathrm{CdTe} / \mathrm{MnTe}$, in $\mathrm{ZnTe} / \mathrm{MnTe}$ the MnTe layers are compressed because $a_{\mathrm{ZnTe}}<a_{\mathrm{MnTe}}$. Such a distortion does not change the type III AFM order [7, 8]. The coupling in ZnTe/MnTe shows an unusual temperature behavior, not seen in CdTe/MnTe. At low temperatures the system produces a magnetic diffraction profile of the type displayed in Fig. 2a (with a central line and symmetrically positioned "satellites"). With increasing $T$, however, it gradually changes into a spectrum of the type depicted in Fig. 2b, with an intensity minimum at the previous central line location. The mechanism responsible for this peculiar behavior is not yet clearly understood.

\subsection{EuTe and EuS based systems}

EuTe/PbTe is another AFM semiconductor superlattice system that has been thoroughly studied by neutron diffractometry. $\left[(\mathrm{EuTe})_{m} \mid(\mathrm{PbTe})_{n}\right]_{N}$ samples with [111] growth axis prepared by $\mathrm{MBE}$ on $\mathrm{BaF}_{2}$ substrates are of remarkably good crystalline quality [20]. EuTe is an fcc antiferromagnet with $T_{\mathrm{N}}=9.6 \mathrm{~K}$. The Eu spins are arranged into ferromagnetic "sheets" on (111)-type planes, and these sheets are antiferromagnetically coupled to one another (the arrangement of spins in the (111) EuTe/PbTe systems closely resembles the model situation depicted in Fig. 1b).

Neutron diffraction studies $[9,10,21]$, performed on a large number $(\approx 50)$ of specimens with many different combinations of $m$ and $n$, have revealed distinct interlayer correlation satellites in samples with $n$ up to 20 . It shows that the interaction between adjacent EuTe can be transferred across non-magnetic PbTe spacers as thick as $70 \AA$. As can be seen in Fig. 4, with increasing $n$ the satellite peaks become less sharp, while a pronounced "hump" appears underneath; the initial set of well-resolved lines gradually changes into the characteristic smooth 


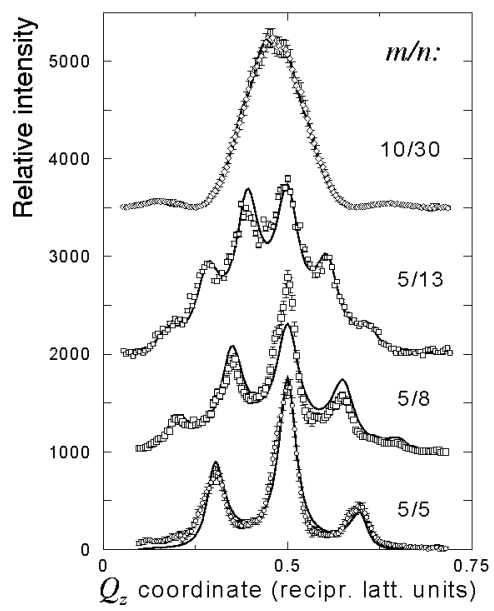

Fig. 4. Measured magnetic diffraction peak profiles from several $\left[(\mathrm{EuTe})_{m} \mid(\mathrm{MnTe})_{n}\right]_{N}$ superlattice samples, illustrating a gradual transition from strong interlayer coupling for smaller $n$ values to an almost completely uncorrelated state in a system with $n=30$. The solid curves are fits of calculated profiles obtained from the "partial correlation" model (see text).

profile of the $\left|F_{\mathrm{s} \text {.l. }}\left(Q_{z}\right)\right|^{2}$ function. This process indicates that - not surprisingly the interlayer correlations gradually weaken with increasing PbTe thickness. The change of the spectrum shape can be described using a phenomenological "partial correlation" model, presented in greater detail in Refs. [22]. Yet, this model does not explain the physical mechanism responsible for the coupling. Because the carrier concentration in the EuTe/PbTe system is several orders of magnitude lower than in metals $\left(10^{16}-10^{17} \mathrm{~cm}^{-3}\right)$, the observed coupling cannot be attributed to Ruderman-Kittel-Kasuya-Yoshida (RKKY) interaction or any other carrier-assisted mechanism. It should also be noted that the coupling model proposed by Rusin [19] for the CdTe/MnTe system cannot be applied to EuTe/PbTe because there are no donor centers of the right type in $\mathrm{PbTe}$. An interesting new development in the problem is a recent work by Blinowski and Kacman (B-K) [23] who explored the possibility that the interlayer interactions are conveyed by valence electronic states in $\mathrm{PbTe}$. Some of the $\mathrm{B}-\mathrm{K}$ model results appear to be in a good qualitative agreement with the experimental data - e.g., the theory predicts that the coupling strength is a relatively slowly decreasing function of the $\mathrm{PbTe}$ spacer thickness, thus explaining the long range of the observed coupling. A more rigorous testing of the $\mathrm{B}-\mathrm{K}$ theory based on the existing results is not yet possible because neutron data alone do not offer quantitative information about the interlayer coupling strength in AFM superlattices.

EuS/PbS is another SL structure based on the Eu chalcogenides that has been investigated by neutron tools $[11,12]$. EuS is an fcc ferromagnet with $T_{\mathrm{c}}=$ 
16.6 K. The SL specimens were prepared on $\mathrm{KCl}$ substrates with a [001] growth axis. Diffraction scans carried out at low temperatures revealed magnetic spectra with a characteristic double-peak profile, a clear signature of antiferromagnetic coupling between the FM layers (see Fig. 5a). This AFM interlayer coupling showed up even more clearly in reflectivity spectra (Fig. 5b) which exhibited sizable maxima at positions corresponding to the doubled structural periodicity of the measured specimen. Such peaks were observed for systems with the non-magnetic spacer thickness $D_{\mathrm{PbS}}$ up to $90 \AA$.

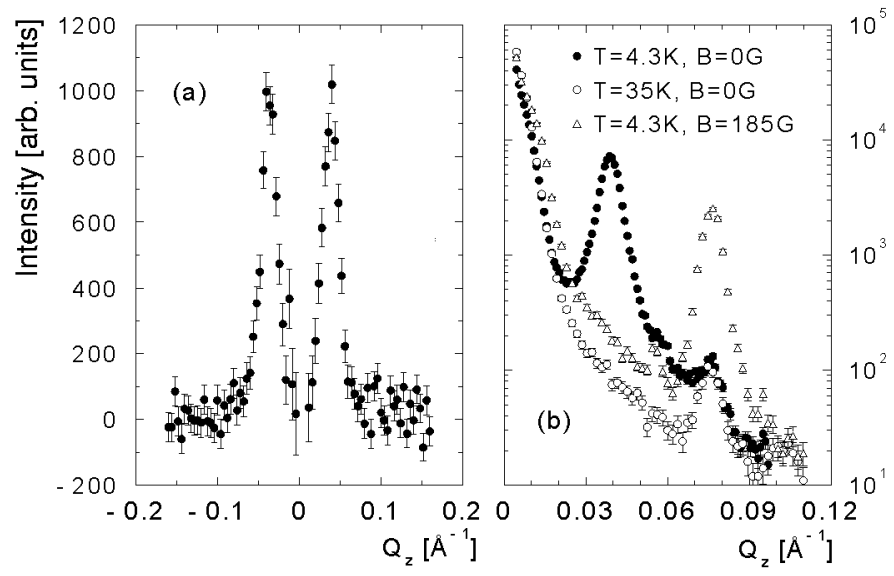

Fig. 5. (a) Diffraction spectrum from an EuS/PbS SL specimen with a $(60 \AA / 23 \AA) \times 15$ composition. Purely magnetic contribution obtained by subtracting data sets taken below and above $T_{\mathrm{C}}$. The characteristic double-peaked profile is a clear signature of AFM coupling between the EuS layers (after [11]); (b) Reflectivity data from the same EuS/PbS specimen. Open circles show the spectrum measured above the Curie point, with a small structural peak corresponding to the SL periodicity. The spectrum taken below $T_{\mathrm{C}}$ and at zero external field (filled circles) exhibits an additional large peak corresponding to doubled SL periodicity arising from AFM coupling between the EuS layers. An external field of 185 Gs enforces a transition to a FM configuration, so the magnetic peak shifts to the structural position (triangles).

The fact that the FM layers have a net magnetic moment makes it possible to manipulate the arrangement of layers in the SL chain. A sufficiently strong external magnetic field enforces a transition to an FM sequence. For $D_{\mathrm{PbS}}$ up to about $20 \AA$, this process was found to be reversible. In this region, the field $B_{\text {sat }}$ needed to attain a full AFM $\rightarrow$ FM transition provides a direct measure of the interlayer coupling strength. For thicker spacers the EuS layers do not return to the AFM configuration even after removing the field, evidently locked in the FM positions by magnetic anisotropy. Here the $B_{\text {sat }}$ value reflects the anisotropy magnitude rather than the coupling strength. 
Model studies on EuS/PbS were performed by Blinowski and Kacman using the same approach as in the earlier work on the EuTe/PbTe system [12]. Since the atomic monolayers in SLs with [001] growth axis consist of both anions and cations (in contrast to the situation in the [111] EuTe/PbTe structures which consist of alternating anion-only and cation-only monolayers), a more sophisticated 3D model version had to be constructed than the previously used $1 \mathrm{D}$ chain. From the calculations it was obtained that an AFM alignment of layers always leads to lower energy than the FM one. This is indeed consistent with the results of measurements on samples with spacer thicknesses from 4 to $90 \AA$, which all show AFM interlayer correlations (experiments for larger spacer thicknesses have yet to be performed). The calculated coupling strength decreases with the $\mathrm{PbS}$ spacer thickness roughly like $2^{-n}$. Such a fast decrease is also consistent with the observations. The model also passes favorably a basic quantitative test, as the calculated coupling energy values are of the same order of magnitude as the values determined from the observed saturation fields $B_{\text {sat }}$.

\subsection{Ga(Mn)As/GaAs superlattices}

The newly synthesized FM semiconductor Ga(Mn)As is the object of great current interest as a prototypic material for developing "spintronics" (i.e., spin-polarized electronics). The material is also highly interesting from the viewpoint of fundamental magnetic studies - a novel aspect is that here the FM interactions between the atomic moments are mediated by holes, not electrons, as is the case in practically all previously known systems exhibiting carrier-mediated magnetism. Insight into the basic magnetic properties of $\mathrm{Ga}(\mathrm{Mn}) \mathrm{As}$ is therefore of considerable current importance. Although the ferromagnetism in $\mathrm{Ga}(\mathrm{Mn}) \mathrm{As}$ has been extensively studied by macroscopic techniques, such data still do not provide conclusive information about the range of the FM ordering. Because of the relatively low concentration of the magnetic ions (up to $7 \%$ ), one can suspect that the ferromagnetism is actually of a "spin-glass type" — or that it consists of very small microdomains. From the viewpoint of spintronics application such a nature of the $\mathrm{Ga}(\mathrm{Mn})$ As ferromagnetism would be a serious complication, since the most desirable situation is that the material spontaneously forms a single-domain F M state, thus reducing the need of external magnetic field. Insight into this issue can be obtained only by methods capable of probing the magnetic correlation on a microscopic level. Neutron scattering tools seem to be extremely well suited for that purpose. However, since $\mathrm{Ga}(\mathrm{Mn}) \mathrm{As}$ is available only in the form of extremely thin epitaxial films or multilayers, the application of conventional "wide-angle" diffractometry for studying this FM system is not possible, because the magnetic diffraction peaks occur at the same positions as the several orders of magnitude stronger Bragg reflections from the GaAs substrate. The substrate reflection problem can be eliminated by growing the samples in the superlattice form of $\mathrm{Ga}(\mathrm{Mn}) \mathrm{As} / \mathrm{GaAs}$ superlattices and using the technique of neutron reflectometry. Yet, reflectometry 
studies using an unpolarized neutron beam (as was the case in experiments on the EuS/PbS system discussed above) appear to be still quite challenging as the magnetic contribution to the reflectivity maxima is still much weaker (10-20\%) than the nuclear contribution. However, the application of the sophisticated technique of polarization-analysis neutron reflectometry makes possible a nearly total separation of the magnetic and nuclear components. Examples of polarization-analysis reflectivity data from a $\mathrm{Ga}(\mathrm{Mn}) \mathrm{As} / \mathrm{GaAs} \mathrm{SL}$ specimen obtained in a recent study [14] are shown in Fig. 6. Each part in the figure shows the results of scans through the first reflectivity Bragg peak for all four combinations of the "spin-up" $(+)$ and "spin-down" (-) states of the incident and the scattered neutron beam.

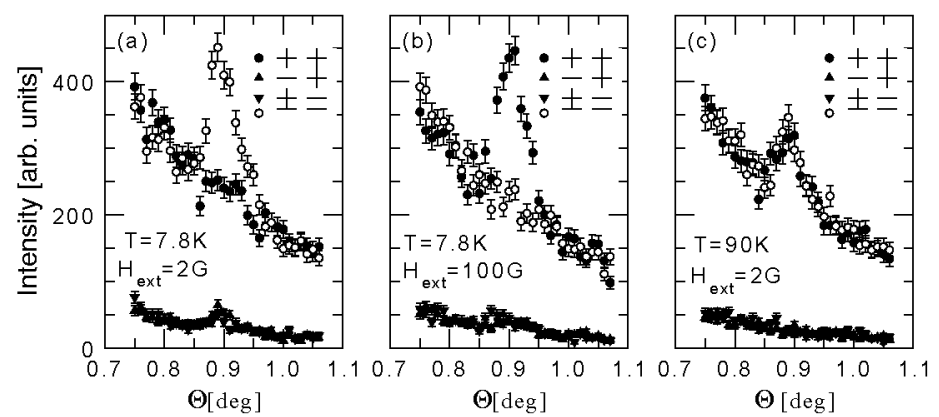

Fig. 6. Polarized neutron reflectometry scans through the first Bragg peak for the $\left[\left(\mathrm{Ga}_{0.94} \mathrm{Mn}_{0.06} \mathrm{As}\right)_{50} \mid(\mathrm{GaAs})_{6}\right]_{50} \mathrm{SL}$ specimen. Four sets of data point in each part show the spectra obtained for four combinations of the spin polarization of the incident and the reflected beam (see text). Part (c) shows data obtained above the Curie temperature. Data in part (a) were recorded after cooling the sample in zero external field (during the measurements the sample was exposed to an upward-oriented 2 Gs stray guide field). The peak in the (--) mode indicates that the sample is magnetized downward. After applying an upward-oriented 100 Gs external field (part (b)), the magnetization, as expected, changes its direction.

In the "spin-flip" (SF) modes, $(+-)$ or $(-+)$, one measures purely magnetic scattering associated with a horizontal magnetization component. In the non-spin-flip (NSF) modes, $(++)$ and (--), the total scattering amplitude is, respectively, the difference and the sum of the nuclear scattering amplitude and the magnetic scattering amplitude due to the vertical magnetization component. It should be noted that in the system studied the sum of the nuclear and magnetic scattering amplitudes for saturated magnetization is nearly equal to the scattering amplitude of pure GaAs. Hence, for the sample magnetized in the vertical direction, the scattering contrast nearly disappears for one NSF mode, and is enhanced for the other.

The data in Fig. $6 \mathrm{c}$, taken above the Curie point $\left(T_{\mathrm{C}} \approx 30 \mathrm{~K}\right)$, show peaks only in NSF modes resulting from purely nuclear scattering. After cooling below 
$T_{\mathrm{C}}$ in zero external field the scans (Fig. 6a) shows only a peak in the (--) mode, and only negligible effects in the $(++)$ and the SF modes, indicating that during the cooling process the bulk of the sample became spontaneously magnetized in the downward direction. This result is indeed very important because it proves that:

- the FM order that spontaneously forms in each Ga(Mn)As layer is truly long-range, single-domain state (any spin-glass like or microdomain structure, or a multidomain arrangement would produce peaks in all four modes);

- the $\mathrm{Ga}(\mathrm{Mn})$ As layers are ferromagnetically coupled across the intervening non-magnetic GaAs spacers.

The latter result provides a direct confirmation of the existence of significant interlayer coupling effects in $\mathrm{Ga}(\mathrm{Mn}) \mathrm{As} / \mathrm{GaAs}$ structures, which was first indirectly deduced from SQUID magnetization measurements on trilayers [1].

\section{Concluding remarks}

Neutron diffraction experiments on the MnTe/II-Te and EuTe/PbTe multilayers have demonstrated two important facts: (i) pronounced interlayer magnetic coupling may occur in superlattices made of nearly-insulating semiconductors; (ii) in such systems, coupling between AFM layers is possible.

The model studies discussed in Sec. 3 show that the transfer of interactions without the assistance of carriers may be explained on the grounds of the electronic theory of semiconductor. However, much more theoretical and experimental insight is still needed. The mechanism proposed by Rusin [18] is definitely of great interest because it offers the possibility of controlling the coupling strength. Yet, the model requires testing by additional experiments. The change of interaction sign seen in $\mathrm{ZnTe} / \mathrm{MnTe}$ is indeed extremely intriguing; hopefully a theoretical explanation of this phenomenon will soon emerge. The $\mathrm{Eu}-\mathrm{VI} / \mathrm{Pb}-\mathrm{VI}$ structures are quite attractive from the viewpoint of theoretical analysis because the same formalism is used to the AFM EuTe/PbTe and the FM EuS/PbS. Some model elements can be tested by experiments on EuS/PbS and then used for explaining effects seen in $\mathrm{EuTe} / \mathrm{PbTe}$, for which it is not possible to directly measure the strength of interlayer coupling.

The results of polarized neutron reflectometry studies of $\mathrm{Ga}(\mathrm{Mn}) \mathrm{As} / \mathrm{GaAs}$ SLs clearly demonstrate that neutron tools may offer valuable insight into the microscopic spin structure of epitaxial spintronics materials. Many new materials of this kind (e.g., strongly p-type $\mathrm{Ga}(\mathrm{Mn}) \mathrm{N}$ or $\mathrm{Zn}(\mathrm{Mn}) \mathrm{O}$ ) are expected to emerge in the near future, and neutron tools will certainly be employed for studying their magnetism. 


\section{Acknowledgments}

Studies on Ga(Mn)As/GaAs, MnTe/CdTe, and Eu-based system presented in this paper were supported by NSF DMR-9972586 and NATO PST.CLG 975228 grants.

\section{References}

[1] H. Ohno, Science 281, 951 (1998).

[2] H. Ohno, A. Shen, F. Matsukura, A. Oiva, A. Endo, S. Katsumoto, Y. Iye, Appl. Phys. Lett. 69, 363 (1996).

[3] T. Dietl, H. Ohno, F. Matsukura, J. Ciberd, D. Ferrand, Science 287, 1019 (2000).

[4] P. Bruno, Phys. Rev. B 52, 411 (1995).

[5] B.A. Jones, IBM J. Res. Dev. 42, 25 (1998).

[6] V. Nunez, T.M. Giebultowicz, W. Fashinger, G. Bauer, H. Sitter, J.K. Furdyna, Mater. Res. Soc. Symp. Proc. 376, 589 (1995); V. Nunez, T.M. Giebultowicz, W. Fashinger, G. Bauer, H. Sitter, J. K. Furdyna, J. Magn. Magn. Mater. 140-144, 633 (1995).

[7] J. Lin, J.J. Rhyne, J.K. Furdyna, T.M. Giebultowicz, J. Appl. Phys. 83, 6554 (1998).

[8] L.E. Stumpe, J.J. Rhyne, H. Kaiser, S. Lee, U. Bindley, J.K. Furdyna, J. Appl. Phys. 87, 6460 (2000).

[9] T.M. Giebultowicz, H. Luo, N. Samarth, J.K. Furdyna, V. Nunez, J.J. Rhyne, W. Fashinger, G. Springholz, G. Bauer, H. Sitter, Physica B 198, 163 (1994).

[10] H. Kepa, K.I. Goldman, T.M. Giebultowicz, C.F. Majkrzak, G. Springholz, H. Krenn, S. Holl, F. Schinagl, G. Bauer, Physica E 2, 399 (1998).

[11] H. Kepa, J. Kutner-Pielaszek, A. Twardowski, A.Yu. Sipatov, C.F. Majkrzak, T. Story, R.R. Galazka, T.M. Giebultowicz, J. Magn. Magn. Mater. 226-230, 1795 (2001).

[12] H. Kepa, J. Kutner-Pielaszek, J. Blinowski, A. Twardowski, C.F. Majkrzak, T. Story, P. Kacman, R.R. Galazka, K. Ha, H.J.M. Swagten, W.J.M. de Jonge, A.Yu. Sipatov, T.M. Giebultowicz, Europhys. Lett. 56, 54 (2001).

[13] A. Stachow-Wojcik, T. Story, W. Dobrowolski, M. Arciszewska, R.R. Galazka, W.M. Kreijveld, C.W.H. Swüste, H.J.M. Swagten, W.J.M. de Jonge, A. Twardowski, A.Yu. Sipatov, Phys. Rev. B 60, 15220 (1999).

[14] H. Kepa, J. Kutner-Pielaszek, A. Twardowski, C. F. Majkrzak, J. Sadowski, T. Story, T.M. Giebultowicz, Phys. Rev. B 64, 121302(R) (2001).

[15] G.E. Bacon, Neutron Diffraction, Clarendon Press, Oxford 1975.

[16] C.F. Majkrzak, Physica B 173, 75 (1991); Acta Phys. Pol. A 96, 81 (1999).

[17] T.M. Giebultowicz, N. Samarth, H. Luo, J.K. Furdyna, P. Klosowski, J.J. Rhyne, Phys. Rev. B 46, 12076 (1992).

[18] T.M. Giebultowicz, P. Klosowski, N. Samarth, H. Luo, J.K. Furdyna, J.J. Rhyne, Phys. Rev. B 48, 12817 (1992). 
[19] T.M. Rusin, Phys. Rev. B 58, 2107 (1998).

[20] G. Springholz, G. Baner, Appl. Phys. Lett. 62, 2399 (1993).

[21] H. Kepa, G. Springholz, K.I. Goldman, T.M. Giebultowicz, C.F. Majkrzak, S. Holl, H. Krenn, P. Kacman, J. Blinowski, G. Bauer, in preparation.

[22] K.I. Goldman, Ph.D. thesis, Oregon State University, 1998, unpublished; K.I. Goldman, H. Kepa, T.M. Giebultowicz, S.K. Sinha, Physica B 241-243, 717 (1998).

[23] J. Blinowski, P. Kacman, Phys. Rev. B 64, 045302 (2001); Acta Phys. Pol. A 19, 719 (1997). 